\title{
Diagnosis, treatment and follow-up of 25 patients with melamine-induced kidney stones complicated by acute obstructive renal failure in Beijing Children's Hospital
}

\author{
Qiang Sun • Ying Shen • Ning Sun • Gui Ju Zhang • \\ Zhi Chen • Jian Feng Fan • Li Qun Jia • \\ Hong Zhan Xiao $\cdot$ Xu Ran Li $\cdot$ Birgit Puschner
}

Received: 30 September 2009 /Revised: 30 September 2009 / Accepted: 5 October 2009 /Published online: 21 October 2009

(C) The Author(s) 2009. This article is published with open access at Springerlink.com

\begin{abstract}
A total of 25 Chinese patients aged 6 to 36 months hospitalised at Beijing Children's Hospital due to melamine-induced kidney stones complicated by acute obstructive renal failure in 2008 were included in a study in order to diagnose and treat these special cases more effectively. Feeding history, clinical presentation, ultrasound findings, treatments and effects were summarised. Twelve to seventeen months follow-up was reported also. Ultrasound examination showed that calculi were located at the kidney and ureters. Stones were composed of both uric acid and melamine in a molar ratio of 1.2:1 to 2.1:1. Treatments providing liquid plus alkalisation of urine proved to be effective in helping the patients pass the
\end{abstract}

Q. Sun $\cdot$ Y. Shen $(\bowtie) \cdot$ G. J. Zhang $\cdot$ Z. Chen $\cdot$ J. F. Fan

Department of Nephrology, Beijing Children's Hospital Affiliated

to Capital Medical University,

Beijing, China 100045

e-mail: ying_116sh@sohu.com

N. Sun $\cdot$ X. R. Li

Division of Urology, Department of Pediatric Surgery,

Beijing Children's Hospital Affiliated

to Capital Medical University,

Beijing, China 100045

L. Q. Jia

Department of Ultrasonography, Beijing Children's Hospital

Affiliated to Capital Medical University,

Beijing, China 100045

H. Z. Xiao

Beijing Institute of Microchemistry,

Beijing, China 100091

B. Puschner

Department of Molecular Biosciences,

School of Veterinary Medicine, University of California,

Davis, CA, USA stones. Surgical intervention was needed in severe cases. Renal function returned to normal in all 25 patients after various durations of therapy. Sixty-eight percent of the patients expelled all of the calculi within 3 months, $90 \%$ in 6 months and $95 \%$ in 9 months, without sequelae till now. Melamine-contaminated milk formula can cause kidney stones in infants, which should be diagnosed by feeding history, clinical symptoms and ultrasound examination. Composition of the stones was not only of melamine but also uric acid. Providing liquid orally or intravenously plus alkalisation of urine proved to promote the removal of the stones. Follow-up of 12 to 17 months after discharge showed no sequelae.

Keywords Melamine $\cdot$ Cystoscopy $\cdot$ Calculi $\cdot$ Uric acid

\section{Introduction}

Urinary tract calculi with renal failure are rarely diagnosed in infants. Of all the patients admitted to Beijing Children's Hospital between 2003 and 2007, the incidence of urinary tract stones complicated by renal failure is only 0.05 to $0.45 \%$. In 2008 , a sharp increase of children diagnosed as urinary calculi complicated by acute obstructive renal failure was noticed in Beijing Children's Hospital, the biggest children's hospital in China. All of the admitted children were under the age of 3 years. Melamine contamination of infant formula was identified as the cause of the renal stones [1]. According to a report from the Chinese Ministry of Health in December of 2008, $22,384,000$ examinations were conducted in infants in China with suspected melamine exposure. By the end of November, 294,000 infants were diagnosed as having 
urinary tract stones and 51,900 infants were hospitalised [14]. In Beijing Children's Hospital, a total of 32,530 children were examined by ultrasound. Of all the screened children, $2.9 \%$ had urinary tract calculi, of which $4.2 \%$ were hospitalised, and 25 of the hospitalised children were subsequently diagnosed as urolithiasis with complication of acute obstructive renal failure. All of the 25 children had consumed the same brand of infant formula, Sanlu, which was contaminated with melamine. The aim of the current study was to describe the clinical and laboratory features, the composition of calculi, the treatment and outcome of the 25 inpatients with melamine-induced kidney stones complicated by acute obstructive renal failure.

\section{Subjects and methods}

From March 15th to November 17th in 2008, 25 inpatients diagnosed as urinary tract calculi and acute obstructive renal failure in Beijing Children's Hospital were enrolled in this study. They were aged 6 to 36 months. Seventeen patients were male and eight were female. The following data were collected: clinical signs, estimated consumption of contaminated formula, urinalysis, ultrasonography of the urinary tract, routine serum chemistry and haematology parameters, including serum urea nitrogen and creatinine, as well as treatment regimens, response to the treatments, duration of hospitalisation and outcome at 12 to 17 months follow-up. Removed calculi were qualitatively and quantitatively analysed by high-performance liquid chromatography coupled with mass spectrometry, gas chromatography coupled with mass spectrometry and Fourier transform infrared spectrometry. Three therapeutic regimens were assessed: cystoscopy and retrograde catheterisation, dialysis (peritoneal and haemodialysis), and fluid administration. Patients' renal function was assessed prior to and after the treatments. The main outcome measures were: cure (renal function returned to normal and all urinary tract calculi were removed), effective (renal function returned to normal and urinary tract calculi decreased in size) and ineffective (urinary tract calculi did not decrease in size). Urinalysis and ultrasound results were recorded every month and serum values of blood urea nitrogen (BUN), creatinine and uric acid were recorded every 3 months.

\section{Results}

Patient population and clinical and diagnostic findings

A total of 25 Chinese patients were included in this study (17 boys and eight girls). They were between 6 months and 3 years old, of whom 19 patients were under 1 year and six patients were between 1 and 3 years old. The median duration of exposure to Sanlu infant formula was 9.5 months (range 15 days to 24 months). The median duration of hospitalisation after stone detection was 14 days (range 4 to 60 days). All of the 25 patients had oliguria, anuria or dysuria. Two patients had haematuria, eight had fever and 12 vomitted, plus diarrhoea or other gastrointestinal tract symptoms, e.g. bloating, abdominal pain, anorexia.

Urinary tract stones were identified by ultrasonography in all of the patients. Ultrasound examination showed that the calculi were usually present in the following two appearences: sand-like crystals, either solitary or in clusters, with no shadow or with a comet-tail-like shadow (Fig. 1); or as larger size, clump-like calculi with a light shadow (Fig. 2). Most of the calculi are located in the renal pelvis and calyces, and at the three stegnosis sites of the ureter: the pyeloureteric junction, the site where the ureter spans the iliac blood vessels and the site where the ureter attaches to the bladder.

Renal functions were significantly impaired in all of the patients: BUN ranged from 14.7 to $49.0 \mathrm{mmol} / \mathrm{l}$, creatinine ranged from 93.0 to $908.0 \mu \mathrm{mol} / \mathrm{l}$, serum potassium ranged from 2.94 to $7.91 \mathrm{mmol} / \mathrm{l}$ and serum uric acid ranged from 195.0 to $1,257.0 \mu \mathrm{mol} / \mathrm{l}$ (Table 1$)$.

\section{Composition of the stones}

Toxicologic analysis to determine the composition of seven of the expelled calculi was performed. Both melamine and uric acid (Fig. 3) were detected in all the calculi in a molar ratio of $1: 1.2$ to $1: 2.1$. None of the calculi contained cyanuric acid.

\section{Treatment and response to treatment}

Exposure to the contaminated milk formula was stopped immediately. Correcting electrolyte abnormalities became the first line of treatment. Children with acute obstructive renal failure and oliguria for at least 2 days or anuria for at least 1 day were dialysed until clinical their chemistry parameters returned to reference ranges. Surgical interventions to remove the stones were given to the patients with severe hydronephrosis and to the anuric patients in whom dialysis was ineffective or unsuitable. In our study, 11 patients (44\%) underwent cystoscopy and retrograde catheterisation; seven (28\%) patients were dialysed, of whom six received peritoneal dialysis and one was haemodialysed; and the remaining seven patients $(28 \%)$ received only fluid administration for 4 to 20 days. The solutions of $1-1.4 \%$ sodium bicarbonate were administered intravenously once or twice daily at a dose of $2 \mathrm{ml} / \mathrm{kg}$ to these patients and they were encouraged to drink plain 
Fig. 1 Image of the kidney (ultrasonography): female, 8 months old, right kidney profile. A $0.15-\mathrm{cm}^{2}$ sand-like calculus (marked with + ) at the renal calyx with a comet-like tail

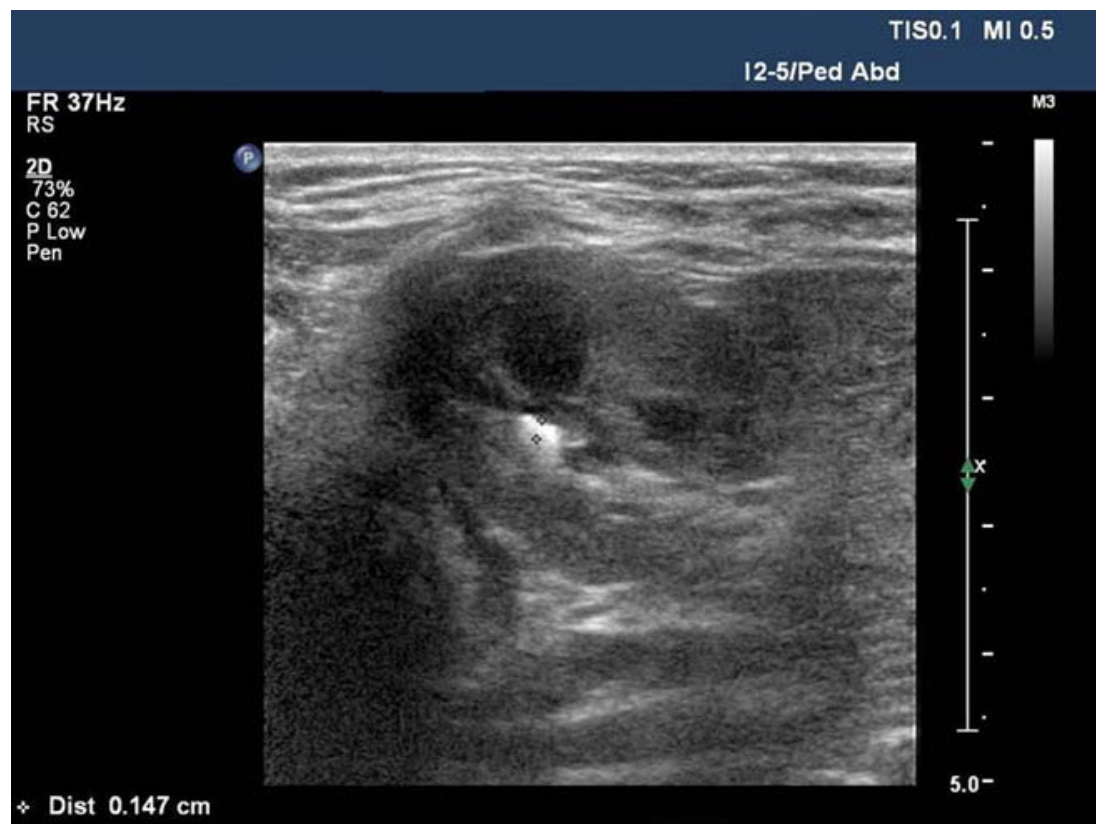

water. Their urine $\mathrm{pH}$ values were continuously monitored for them to reach 6.5-7.0. Before fluid administration, the stone sizes ranged from 0.10 to $1.92 \mathrm{~cm}^{2}$ (by ultrasound), but after alkalisation, they reduced to 0 to $1.72 \mathrm{~cm}^{2}$.

The renal functions returned to normal within 7 days (median 3 days) in all of the patients. Creatinine concentrations decreased to normal concentrations in $3.5 \pm$ 1.89 days in the cystoscopy group, in $3.8 \pm 2.26$ days in the dialysis group and in $2.7 \pm 1.60$ days in the patients receiving fluids. According to the criteria we mentioned earlier in this article, six of the 25 patients were cured and 19 were effective by the time of discharging.
Follow-up

Twenty-two patients were reviewed from May 1st 2008 to September 5th 2009; three patients failed the follow-up because of changes of address. At follow-up visits, all of the 22 patients had normal renal function and normal urine routine. The medium urine $\mathrm{pH}$ was 6.0 . Serum uric acid values were normal. With ultrasonography study, $68 \%$ of the patients were found to have no urinary tract calculi at 3 months follow-up, $90 \%$ at 6 months and $95 \%$ at 9 months, respectively (Fig. 4). Hydronephrosis or other sequelae were not identified. To the two patients who still had calculi
Fig. 2 Image of the kidney (ultrasonography): male, 10 months old, left kidney profile. The $0.6 \times 0.4-\mathrm{cm}^{2}$ calculus at the renal calyx has a light shadow

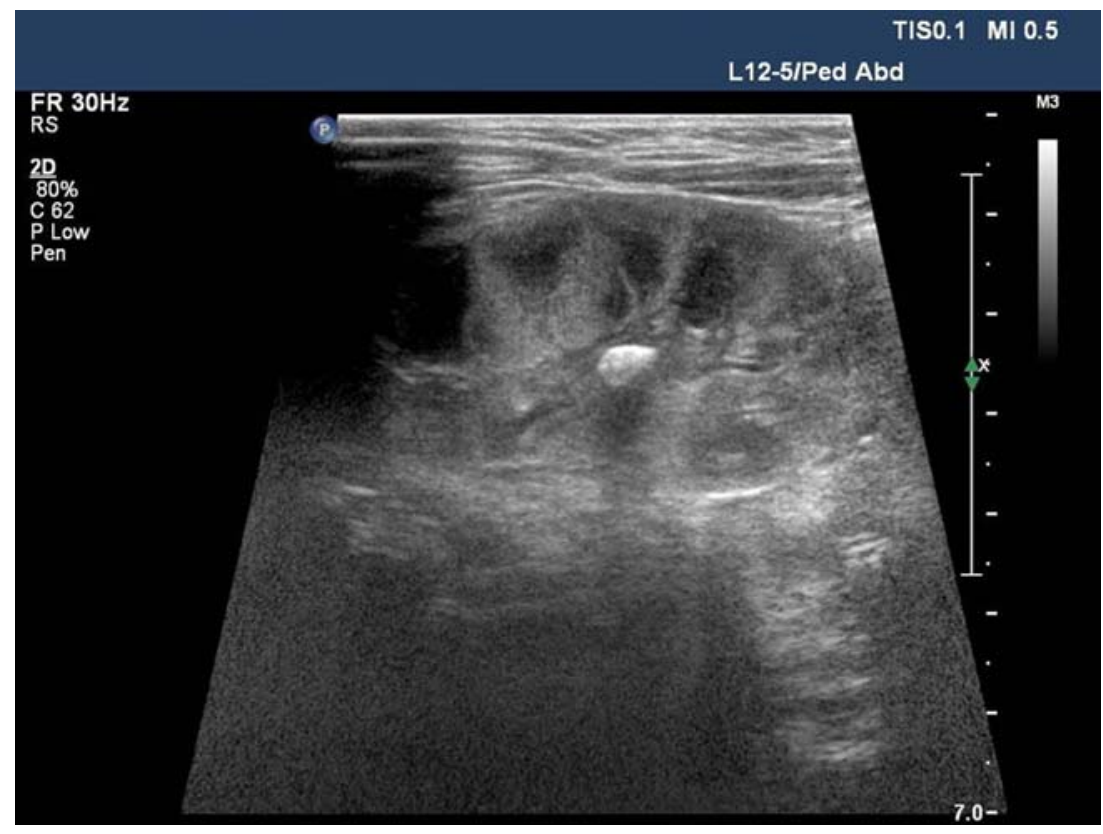




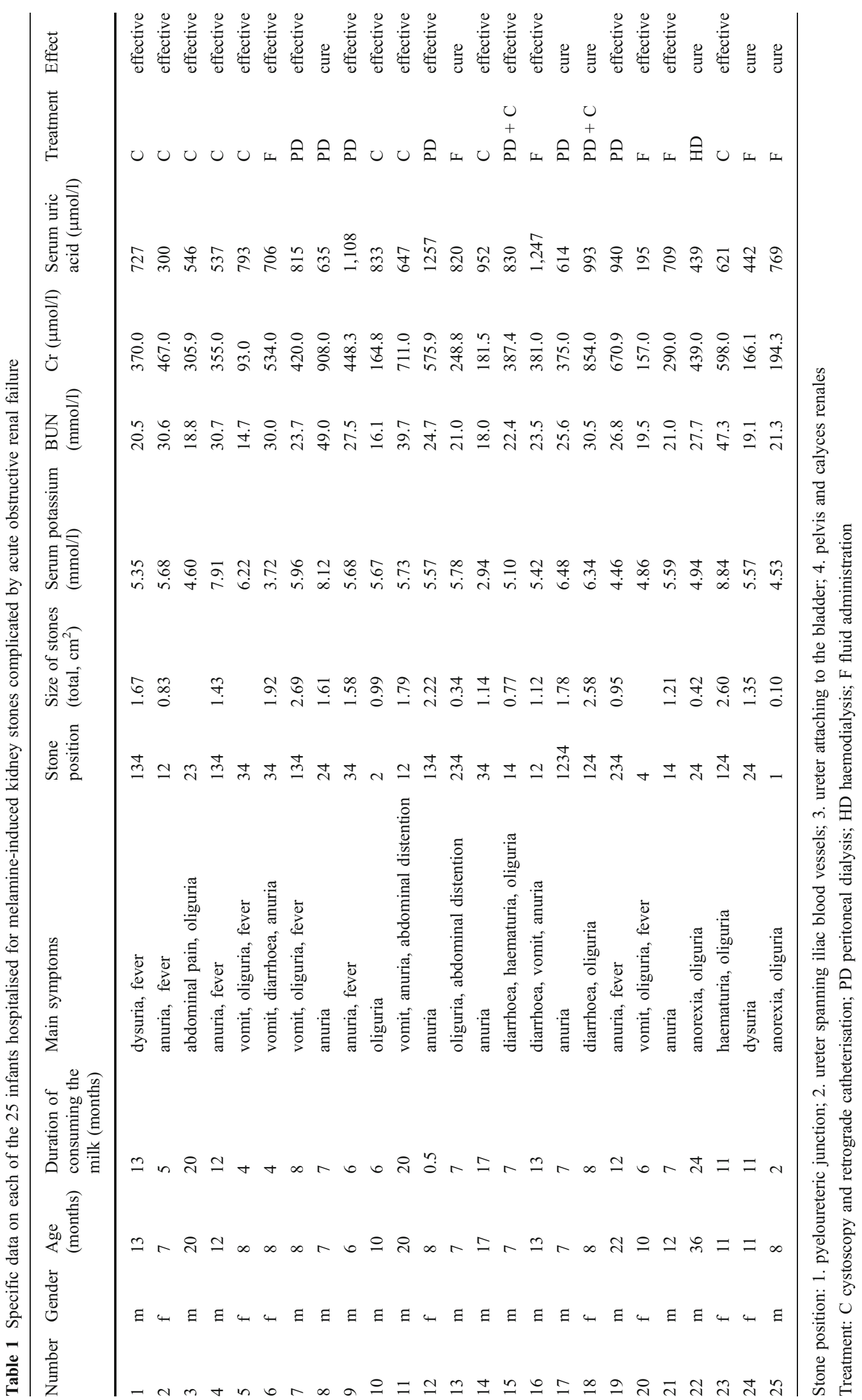




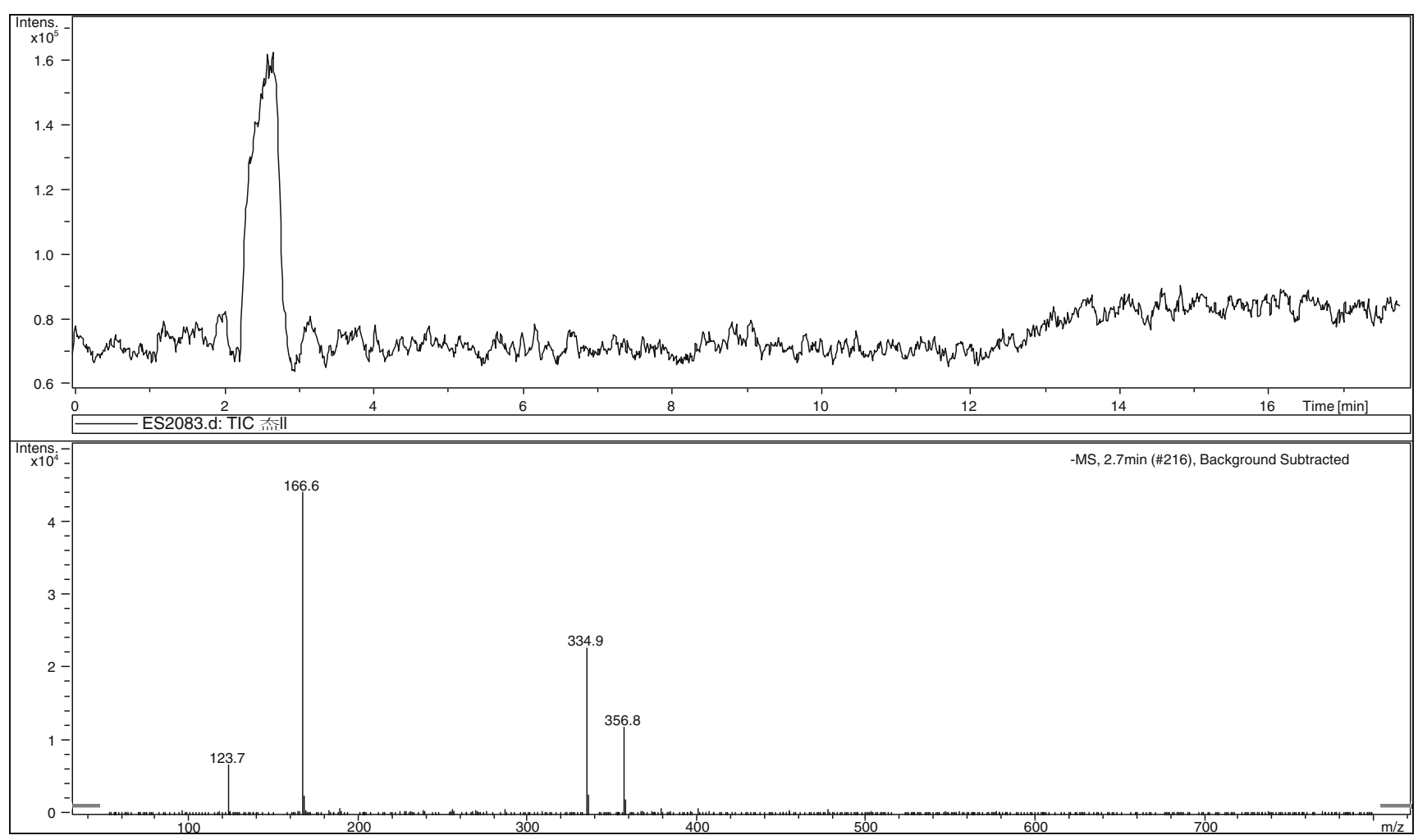

Fig. 3 Total ion chromatography of high-performance liquid chromatography-mass spectrometry (LCMS) and mass spectrogram of uric acid (detected in negative ion mode)

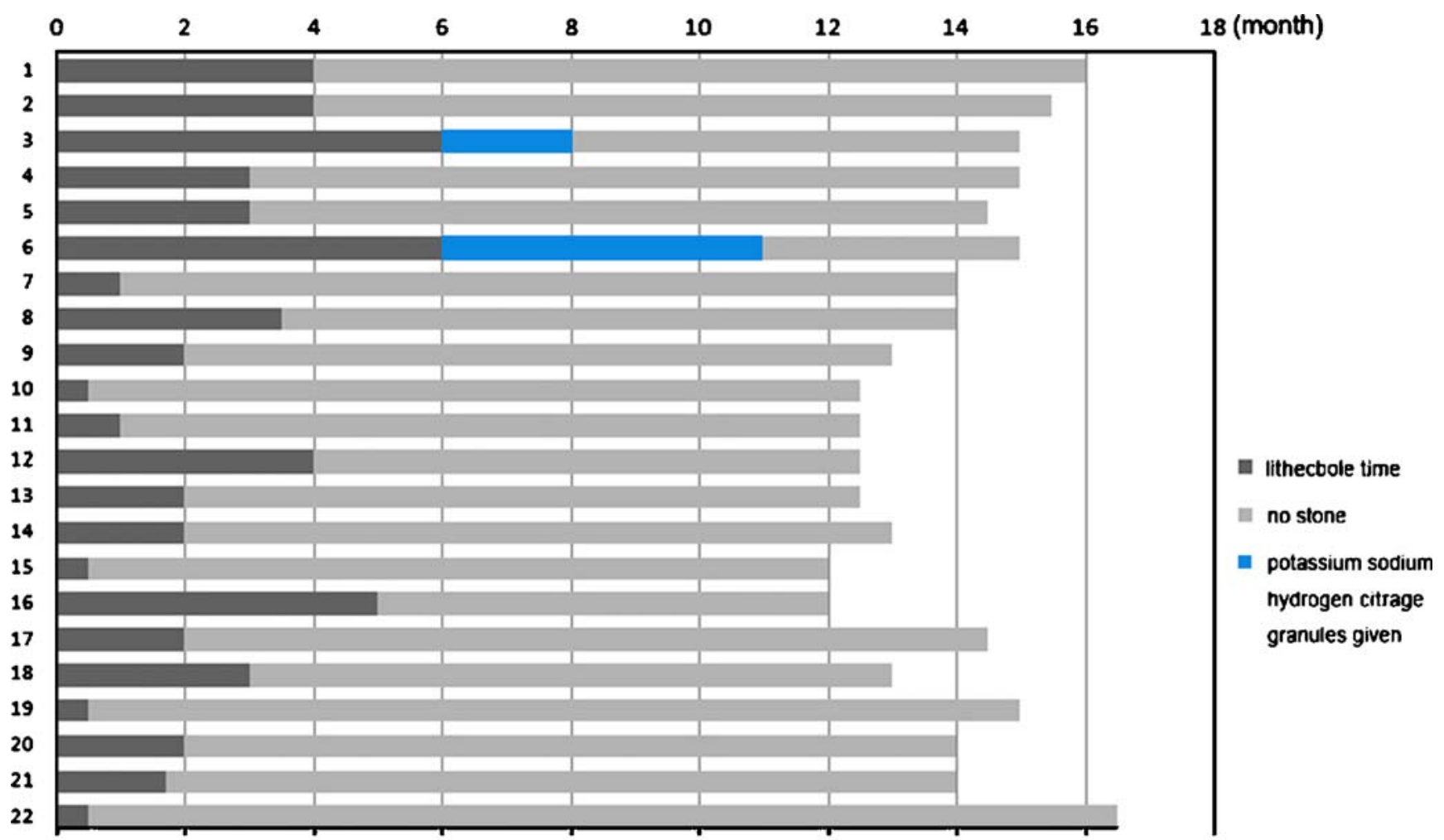

Follow-up of the 22patients

Fig. 4 Follow-up of 22 of the patients 
after 6 months, potassium sodium hydrogen citrate granules ( $1 \mathrm{~g} /$ day, oral) was given. After that, one patient had no stones at 8 months and the other had his calculi decreased in size. The latter was an 8-month-old boy with the largest stone $\left(2.69 \mathrm{~cm}^{2}\right)$. Eleven months after discharge from our hospital, the size of the stone was still quite big $\left(1.20 \mathrm{~cm}^{2}\right)$, then, ultrasound-guided extracorporeal shockwave lithotripsy was given to this patient successfully. Till now (15 months follow-up), no stones were found by urinary tract ultrasonography, and the urinalysis and renal function of the patient remained normal.

\section{Discussion}

Physicians should take into consideration obstructive nephropathy when infants show clinical signs of pain, vomiting, haematuria and the presence of crystals during a urinalysis. The diagnostic approach for infants with suspected melamine-induced kidney stones relies heavily on ultrasonography of the urinary tract because melamineuric acid calculi cannot be detected by radiography. Calculi or crystals have to be at least $1 \mathrm{~mm}$ in diameter in order to be detected by ultrasonography. Intravenous pyelography (IVP), in which calculi manifest as filling defects, may also be used to evaluate renal function, although this technique was not used in these 25 patients. Computerised tomography (CT) did not provide definite evidence for diagnosis, so is not recommended. Accurate diagnosis is the key to successful treatment and outcome, and must include detailed feeding history, clinical examination, imaging data and chemical analysis of urine and calculi [12].

The male:female ratio of the 25 patients was 2.1:1 in this study. This is in agreement with the ratio reported in a study assessing the influence of gender on the development of paediatric renal stones [3]. The anatomical features of the male urethra are different from females in terms of length, stegnosis and arcuations; these differences may predispose male infants to urinary tract calculi because of the larger difficulty in passing stones.

This study demonstrates that no cyanuric acid contributed to the calculus formation in the affected infants and that the calculi consisted of both uric acid and melamine. We postulate that melamine is alkalescent and some forms of melamine can turn into cyanuric acid while contacting strong acid in gastric juice, and cyanuric acid can then lower body fluid $\mathrm{pH}$ values. When $\mathrm{pH}$ values are lower than 5.5, uric acid tends to accumulate and form crystals that can block urine flow in the tubules $[2,4,13]$. The renal failure results from both intrarenal crystal-associated obstruction and an elevation in renal pressure that reduces renal blood flow and glomerular filtration. It is a mechanical obstruction that results in renal damage, rather than a systemic toxic effect $[5,7-10]$.

As urinary $\mathrm{pH}$ played an important role in the development of melamine-uric acid calculus, urine alkalisation with potassium citrate or sodium bicarbonate in our treatment proved to be highly effective, resulting in dissolution of the existing stones $[6,11]$.

Twelve to seventeen months follow-up demonstrated that most of the patients passed all stones within 3 months. When it is difficult to expel stones after 6 months, surgical intervention should be considered to help the patient to get rid of the stones which are large in size $\left(\right.$ e.g. $\left.1 \mathrm{~cm}^{2}\right)$ or located at poor positions, e.g. renal pelvis and calyces renales. One of the 25 patients who did not expel all the stones after 11 months was given ultrasound-guided extracorporeal shockwave lithotripsy and the patient was cured without any complications. Currently, a further follow-up survey for the 25 patients is being carried out by our hospital.

In conclusion, melamine-contaminated formula can cause urinary system calculi and acute obstructive renal failure in infants. The stones are composed of uric acid and melamine at a molar ratio ranging from 1.2:1 to 2.1:1, without evidence of the presence of cyanuric acid or other melamine analogues. Therapeutic interventions have included oral fluid (plain water) administration, intravenous fluid administration and alkalisation of urine (to reach urine $\mathrm{pH}$ values of 6.5-7.0) with bicarbonate. Peritoneal dialysis, haemodialysis and surgical intervention for the removal of the stones were necessary in treating severe cases. The 12 to 17 months follow-up revealed no sequelae, while the longterm prognosis is still unclear and needs further observations.

Acknowledgements The authors would like to acknowledge all of the doctors, nurses and patients participating in this study. We also thank Jinfang Wang and Junlan Lu for their selfless help.

Conflict of interest/disclosure policy Qiang Sun (none), Ying Shen (none), Ning Sun (none), Gui Ju Zhang (none), Zhi Chen (none), Jian Feng Fan (none), Xu Ran Li (none), Li Qun Jia (none), Hong Zhan Xiao (none), Birgit Puschner (none).

Open Access This article is distributed under the terms of the Creative Commons Attribution Noncommercial License which permits any noncommercial use, distribution, and reproduction in any medium, provided the original author(s) and source are credited.

\section{References}

1. Chan EYY, Griffiths SM, Chan CW (2008) Public-health risks of melamine in milk products. Lancet 372:1444-1445

2. Conger JD, Falk SA (1977) Intrarenal dynamics in the pathogenesis and prevention of acute urate nephropathy. J Clin Invest 59:786-793 
3. Coward RJM, Peters CJ, Duffy PG et al (2003) Epidemiology of paediatric renal stone disease in the UK. Arch Dis Child 88:962-965

4. Davidson MB, Thakkar S, Hix JK et al (2004) Pathophysiology, clinical consequences, and treatment of tumor lysis syndrome. Am J Med 116:546-554

5. Dobson RL, Motlagh S, Quijano M et al (2008) Identification and characterization of toxicity of contaminants in pet food leading to an outbreak of renal toxicity in cats and dogs. Toxicol Sci 106:251-262

6. Ferrari P, Bonny O (2004) Diagnosis and prevention of uric acid stones. Ther Umsch 61:571-574

7. González J, Puschner B, Pérez V et al (2009) Nephrotoxicosis in Iberian piglets subsequent to exposure to melamine and derivatives in Spain between 2003 and 2006. J Vet Diagn Invest 21:558-563

8. Kim B, Perkins LB, Bushway RJ et al (2008) Determination of melamine in pet food by enzyme immunoassay, high-performance liquid chromatography with diode array detection, and ultraperformance liquid chromatography with tandem mass spectrometry. J AOAC Int 91:408-413
9. Puschner B, Poppenga RH, Lowenstine LJ et al (2007) Assessment of melamine and cyanuric acid toxicity in cats. J Vet Diagn Invest 19:616-624

10. Reimschuessel R, Gieseker CM, Miller RA et al (2008) Evaluation of the renal effects of experimental feeding of melamine and cyanuric acid to fish and pigs. Am J Vet Res 69:1217-1228

11. Shen Y, Sun N, Jiang YP (2008) Explanation on "the treatment of the urinary calculus of the infant fed with melamine polluted formula milk". Zhonghua Er Ke Za Zhi 46:816-819

12. Sun N, Shen Y, Sun Q et al (2008) Melamine related urinary calculus and acute renal failure in infants. Zhonghua Er Ke Za Zhi 46:810-815

13. Tiu RV, Mountantonakis SE, Dunbar AJ et al (2007) Tumor lysis syndrome. Semin Thromb Hemost 33:397-407

14. World Health Organization (WHO) (2008) Toxicological and health aspects of melamine and cyanuric acid. Report of a WHO Expert Meeting in Collaboration with FAO Supported by Health Canada, 1st-4th December 2008. Available online at: http:// whqlibdoc.who.int/publications/2009/9789241597951_eng.pdf 